Edited by

Lorenzo Manti,

University of Naples Federico II, Italy

Reviewed by

Pankaj Chaudhary,

Queen's University Belfast,

United Kingdom

Edward Graves,

Stanford University, United States

*Correspondence:

Elise Konradsson elise.konradsson@med.lu.se

tThese authors have contributed equally to this work and share first authorship

${ }^{\ddagger}$ These authors have contributed equally to this work and share last authorship

Specialty section: This article was submitted to Radiation Oncology, a section of the journa

Frontiers in Oncology

Received: 24 January 2021 Accepted: 26 April 2021 Published: 13 May 2021

Citation:

Konradsson E, Arendt ML, Bastholm Jensen K, Børresen B, Hansen AE, Bäck S, Kristensen AT, Munck af Rosenschöld P Ceberg C and Petersson K (2021) Establishment and Initial Experience of

Clinical FLASH Radiotherapy

in Canine Cancer Patients.

Front. Oncol. 11:658004. doi: 10.3389/fonc.2021.658004

\section{Establishment and Initial Experience of Clinical FLASH Radiotherapy in Canine Cancer Patients}

\author{
Elise Konradsson ${ }^{1 \star \dagger}$, Maja L. Arendt ${ }^{2 \dagger}$, Kristine Bastholm Jensen ${ }^{3}$, Betina Børresen ${ }^{2}$, \\ Anders E. Hansen ${ }^{4}$, Sven Bäck ${ }^{5}$, Annemarie T. Kristensen ${ }^{2}$, Per Munck af Rosenschöld ${ }^{5}$, \\ Crister Ceberg ${ }^{17}$ and Kristoffer Petersson ${ }^{5,6 \neq}$ \\ ${ }^{1}$ Medical Radiation Physics, Department of Clinical Sciences, Lund University, Lund, Sweden, ${ }^{2}$ Department of Veterinary \\ Clinical Sciences, University of Copenhagen, Frederiksberg, Denmark, ${ }^{3}$ Veterinärhuset Öresund, Limhamn, Sweden, \\ ${ }^{4}$ Department of Biotherapeutic Engineering and Drug Targeting, DTU Health Tech, Kgs, Technical University of Denmark, \\ Lyngby, Denmark, ${ }^{5}$ Radiation Physics, Department of Hematology, Oncology and Radiation Physics, Skåne University \\ Hospital, Lund, Sweden, ${ }^{6}$ Department of Oncology, Oxford Institute for Radiation Oncology, University of Oxford, \\ Oxford, United Kingdom
}

FLASH radiotherapy has emerged as a treatment technique with great potential to increase the differential effect between normal tissue toxicity and tumor response compared to conventional radiotherapy. To evaluate the feasibility of FLASH radiotherapy in a relevant clinical setting, we have commenced a feasibility and safety study of FLASH radiotherapy in canine cancer patients with spontaneous superficial solid tumors or microscopic residual disease, using the electron beam of our modified clinical linear accelerator. The setup for FLASH radiotherapy was established using a short electron applicator with a nominal source-to-surface distance of $70 \mathrm{~cm}$ and custom-made Cerrobend blocks for collimation. The beam was characterized by measuring dose profiles and depth dose curves for various field sizes. Ten canine cancer patients were included in this initial study; seven patients with nine solid superficial tumors and three patients with microscopic disease. The administered dose ranged from 15 to $35 \mathrm{~Gy}$. To ensure correct delivery of the prescribed dose, film measurements were performed prior to and during treatment, and a Farmer-type ion-chamber was used for monitoring. Treatments were found to be feasible, with partial response, complete response or stable disease recorded in 11/13 irradiated tumors. Adverse events observed at follow-up ranging from 3-6 months were mild and consisted of local alopecia, leukotricia, dry desquamation, mild erythema or swelling. One patient receiving a 35 Gy dose to the nasal planum, had a grade 3 skin adverse event. Dosimetric procedures, safety and an efficient clincal workflow for FLASH radiotherapy was established. The experience from this initial study will be used as a basis for a veterinary phase $1 / / I$ clinical trial with more specific patient inclusion selection, and subsequently for human trials.

Keywords: flash, ultra-high dose rate, radiotherapy, radiation oncology, canine cancer patients, normal tissue, dosimetry 


\section{INTRODUCTION}

FLASH radiotherapy (FLASH-RT) has emerged as a treatment modality with the potential to revolutionize the field of radiotherapy. The radiation dose is delivered in a fraction of a second, which is considerably faster than conventional radiotherapy, where the dose rate is typically a few Gy per minute. In 2014, Favaudon et al. presented the concept of FLASH (1), showing that delivering the dose at ultra-high dose rates resulted in reduced normal tissue toxicity in mice compared to delivering the dose at conventional dose rates, while being equally effective in killing cancer cells. Since then, several in vivo studies have been conducted confirming the sparing effect (2-7) and the retained tumor control (6-8). One veterinary trial has been published on FLASH-RT, including six feline cancer patients with squamous cell carcinoma of the nasal planum, also proving the potential of this technique (5). So far, one human treatment has been reported, with promising results (9). This patient suffered from a CD30+ T-cell cutaneous lymphoma and the treatment was administered as a single fraction of $15 \mathrm{~Gy}$.

These intriguing findings have resulted in an increased interest in advancing FLASH-RT towards clinical trials $(7,10$, 11). The progression towards this goal has been limited by the low availability of accelerators that can deliver ultra-high dose rate electrons in a clinical setting. Most of the studies mentioned above, including the first human treatment, have been conducted in research environments intended for preclinical experiments, with accelerators that are not designed for medical use (1-5, 7-9, 12). However, it has recently been shown, by our group and others, that clinical linear accelerators can be modified to deliver the dose rates needed to observe a FLASH effect (13-15). Our group has modified an Elekta Precise linear accelerator (14) so that it can operate at dose rates of 400-500 Gy/s at a source-tosurface distance of $70 \mathrm{~cm}$. The possibility to perform FLASH studies using clinical linear accelerators opens up for more widespread research in this area, and facilitates translation into clinical studies.

To further explore the potential of FLASH-RT, a feasibility and safety study of FLASH-RT in canine cancer patients with spontaneous superficial solid tumors or microscopic residual disease using the electron beam of our modified clinical linear accelerator was initiated. Radiotherapy in canine cancer patients is well documented as a standard of care treatment modality for multiple tumor pathologies (16). However, in Europe, radiotherapy is used less commonly for treatment of veterinary cancer patients compared to the human situation, mainly due to lack of availability, cost and the need for multiple anesthesias required for conventional fractionated radiotherapy. Companion animal cancers are comparable to their human counterparts. They develop spontaneously in an immune competent host, at similar sizes, types, biological environment, and with similar clinical approaches to diagnosis and treatment modalities used $(17,18)$. This allows for veterinary clinical trials with similar radiation qualities, field sizes and targets as for human patients. Therefore, companion animal cancer patients provide an opportunity for performing cross-disciplinary research that has the potential to benefit human and veterinary cancer patients alike. In a recent review article by Nolan et al., the authors describe previous translational studies, where canine cancer patients have been used to model normal tissue response, tumor oxygenation and DNA damage response, and to optimize irradiation parameters for human radiotherapy (19). Companion animal cancer patients are usually treated with radiotherapy over a period 2-4 weeks, typically 16-20 fractions with a fractional dose of 2.5-4 Gy (19), requiring multiple anesthesia sessions which may be stressful for the patient. In contrast, FLASH-RT is delivered in a single or a few fraction(s), making this modality practical and attractive for companion animal radiotherapy. Although, previous preclinical studies have shown that fractionation of FLASH treatment does not negatively affect tumor control, some have indicated that the normal tissue sparing of FLASH is lost for fractionated treatment, where the fraction dose is below $10 \mathrm{~Gy}(7,8)$. For these reasons, the canine cancer patients included in the current study received single fraction FLASH-RT, a treatment modality not otherwise available to them, which also provided us important data not attainable in preclinical rodent models.

In this paper the establishment of a clinical workflow for electron FLASH-RT in canine cancer patients is presented, with the initial overall aim of describing dosimetric procedure, treatment parameters, possible adverse events and treatment responses. This is an important step in the development of a safe and efficient workflow for FLASH-RT in a clinical setting, which could inform future human clinical trials.

\section{MATERIALS AND METHODS}

\section{Irradiation Source}

The irradiation source was a clinical Elekta Precise linear accelerator (Elekta $\mathrm{AB}$, Stockholm, Sweden) with Integrity software version 1.2 temporarily modified for electron FLASH irradiation, previously described by Lempart et al. (14). The accelerator could be modified for FLASH delivery and switched back to clinical mode in a few minutes. To achieve maximal radiation output the accelerator was operated with increased electron gun filament current and without primary and secondary scattering foils. The radiation was delivered with the standard pulse structure of $3.5 \mu$ sulses at a pulse repetition frequency of $200 \mathrm{~Hz}$. To allow the accelerator to be controlled on a pulse-by-pulse basis, an in-house built electronic circuit and a microcontroller unit was used with a diode as a beam pulse detector. Due to slight day-to-day variations, the gun filament current and magneton frequency needed to be manually tuned to achieve maximum output. This was facilitated by relative measurements with an ion-chamber.

\section{Setup, Beam Characterization, and Dosimetric Procedure}

A setup for FLASH-RT in companion animals was established using an electron applicator with a nominal source-to-applicator distance of $65 \mathrm{~cm}$. For practical reasons, the source-to-surface 
distance was fixed at $70 \mathrm{~cm}$, i.e. $5 \mathrm{~cm}$ distance from the distal edge of the electron applicator. Custom-made Cerrobend blocks of various sizes were created and attached to the end of the electron applicator for field collimation. To characterize the beam, dose profiles at $2 \mathrm{~cm}$ depth and depth dose curves (0-4.2 cm depth) were measured in a Solid Water HE phantom (Gammex Inc., Middleton, Wisconsin, USA) using dose rate independent (20) radiochromic film (GafChromic EBT-XD, Ashland Specialty Ingredients G.P., Bridgewater, New Jersey, USA). The radiochromic film batch was calibrated in a clinical 10 $\mathrm{MeV}$ beam, against an ion-chamber traceable to a standard laboratory for a dose range of 1-40 Gy. Dose maximum, halfvalue depth, therapeutic range, full width at half maximum (FWHM) and penumbra widths (80\%-20\%) were determined for each given field size.

Prior to each treatment, film measurements were performed in phantoms mimicking the treatment geometry (Figure 1) to determine the total dose as well as the dose-per-pulse (DPP) and number of pulses to be delivered to the given patient. These measurements were related to the signal from a Farmer-type ionchamber (NE 2505/3-3A) positioned in a custom-made holder in the applicator. During treatment, the Farmer-type ion-chamber was used as an on-line monitor. In addition, EBT-XD film was used for in vivo dose measurements at the skin surface in the center of the beam to verify the delivered dose (Figure 2). The treatment volumes ( $\geq 80 \%$ of the prescribed dose) were estimated based on vertical film measurements in the solid water phantom.

\section{Canine Cancer Patients}

The patients enrolled in this study were diagnosed during routine work up and staging with superficial solid cancers such as carcinomas, sarcomas, mast cell tumors and malignant melanomas or post-operative microscopic residual disease, where radiotherapy is the standard of care or only treatment
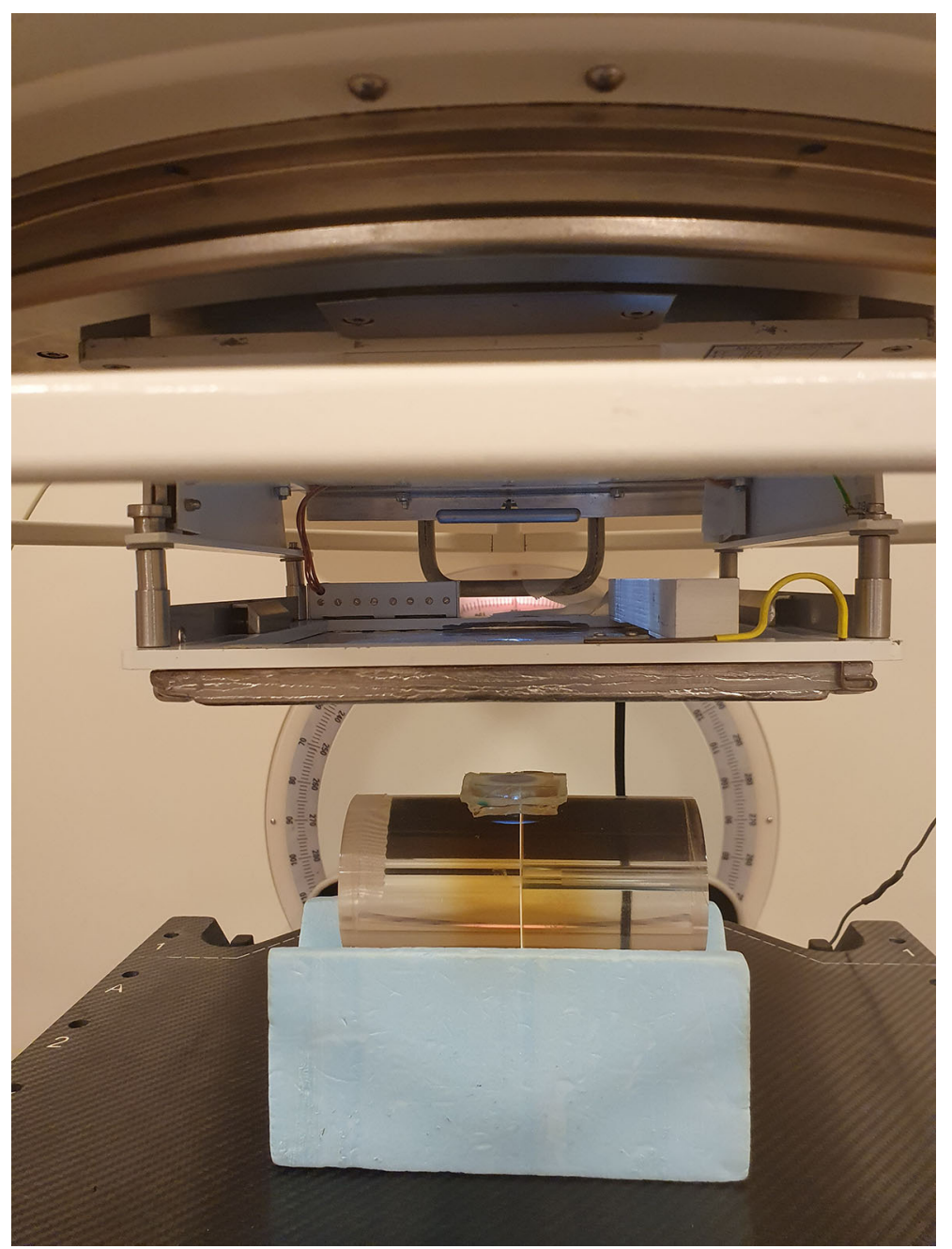

FIGURE 1 | In preparation for each patient treatment, measurements with radiochromic film were performed in phantoms mimicking the treatment geometry, to determine the total dose as well as the dose-per-pulse and number of pulses to be delivered to the patient. A Farmer-type ion-chamber positioned in a customized holder in the electron applicator was used as an output monitor. 

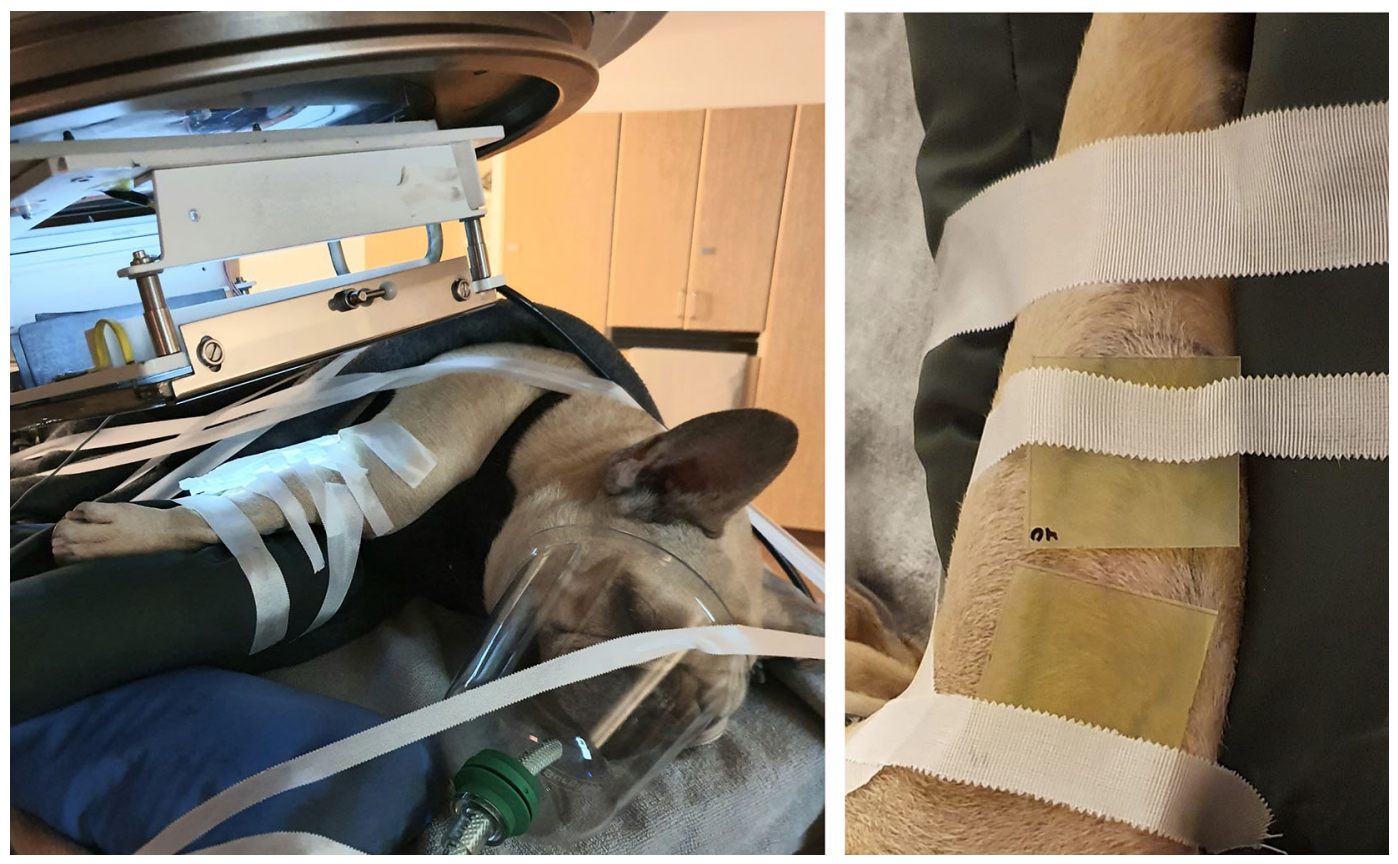

FIGURE 2 | The treatment setup for patient no. 1 (left panel), with a source-to-surface distance of $70 \mathrm{~cm}$ and a Cerrobend plate to collimate the $8 \times 4 \mathrm{~cm}^{2}$ radiation field. During the treatment, in vivo film measurements (right panel) were performed at the skin surface of the patient to verify the delivered dose.

alternative or where the owners had declined other treatment options. Diagnosis was confirmed with histopathology. Patients evaluated as poor candidates for anesthesia, such as patients with hepatic or renal insufficiency or severe heart disease, were excluded from the trial. This initial clinical feasibility and safety study included ten canine cancer patients; seven patients with a total of nine solid tumors and three patients with microscopic residual disease (Table 1).

\section{Study Design}

This clinical feasibility and safety study was designed as a doseescalation trial, starting at a dose level of 15 Gy. Two-three patients were included at each dose level. A dose escalation of 5 Gy was successively performed provided no grade 3 toxicities were observed. Further consideration of different normal and tumor tissues' sensitivity to radiotherapy was taken into account in the prescription. The primary endpoint was to evaluate the feasibility and safety of FLASH-RT with this setup, and thus this initial study was not designed to provide statistically valid results of tumor response following FLASH-RT. Though not a primary purpose of the study, a clinical benefit to the patients treated in the study was also expected.

\section{FLASH-RT}

The ten canine cancer patients were treated during the period from March to November 2020. All tumor sites received a single beam single fraction of FLASH-RT, except for one tumor (patient no. 5) which was re-irradiated one month after the first treatment.
To improve the dose distribution, treatments were planned in terms of field size and bolus thickness based on 1) clinical examination and caliper measurements, 2) CT images and/or photographs of the tumor, and 3) the beam characteristics. Tissue equivalent bolus material (Elasto-Gel EP Padding, Southwest Technologies, North Kansas City, Missouri, USA) was used for some treatments to reduce the treatment depth of the electron beam and to increase the surface dose. For oral tumors and tumors of the eyelid, an internal lead shield was used as a beam stopper to protect normal tissue. Treatment margins of 5-10 mm was used for solid tumors, and 10-20 mm for surgical scars. Setup and treatment angle was determined when the patient was positioned on the treatment couch. Prior to treatment, patients were sedated using an adapted protocol, which enable recovery within minutes after completion of the treatment. The radiation dose was prescribed at the depth of dose maximum and was decided through discussion between medical physicists and board-certified veterinary oncologists based on tumor type and any adverse events observed in previous treatments.

\section{Follow-Up Procedure}

Follow-up clinical evaluation occurred at approximately 7 days, 1 month and 3 months post FLASH-RT. At each follow-up evaluation, tumor response or signs of progression or relapse was evaluated together with evidence of local radiation adverse events. Tumor response was estimated based on the veterinary RECIST 1.0 criteria for patients with solid tumors (21), and disease-free interval was calculated for patients with microscopic 
TABLE 1 | Description of the ten canine patients.

\begin{tabular}{|c|c|c|c|c|c|c|c|c|}
\hline $\begin{array}{l}\text { Patient } \\
\text { no. }\end{array}$ & Breed & $\begin{array}{c}\text { Age } \\
\text { [years] }\end{array}$ & $\begin{array}{l}\text { Weight } \\
\text { [kg] }\end{array}$ & Target site & Diagnosis & State of disease & $\begin{array}{l}\text { Post-op } \\
\text { RT? }\end{array}$ & $\begin{array}{l}\text { Est. gross tumor volume } \\
\qquad\left[\mathrm{cm}^{3}\right]\end{array}$ \\
\hline 1 & French Bulldog & 7 & 13.9 & Front limb & Soft tissue sarcoma & Grade 1 & Yes & $\mathrm{n} / \mathrm{a}$ \\
\hline 2 & Xoloitzcuintle & 10 & 10.3 & Front limb & Soft tissue sarcoma & Grade 1 & Yes & $\mathrm{n} / \mathrm{a}$ \\
\hline 3 & French Bulldog & 7 & 12.7 & Front limb & Mast cell tumor & Grade 2 & Yes & $\mathrm{n} / \mathrm{a}$ \\
\hline \multirow[t]{2}{*}{4} & Sibirian Husky & 11 & 15.9 & Hind limb & Plasmacytoma & $\mathrm{n} / \mathrm{a}$ & No & 1.3 \\
\hline & & & & Hind limb & Soft tissue sarcoma & Unknown & No & 11 \\
\hline \multirow[t]{2}{*}{5} & $\begin{array}{l}\text { Labrador } \\
\text { Retriever }\end{array}$ & 11 & 42.0 & Eyelid & Melanoma & $\begin{array}{l}\text { Amelanotic } \\
\text { malignant }\end{array}$ & Yes $^{\dagger}$ & 0.3 \\
\hline & & & & Eyelid & Melanoma & $\begin{array}{l}\text { Amelanotic } \\
\text { malignant }\end{array}$ & Yes & 0.5 \\
\hline 6 & Border Collie & 13 & 9.7 & $\begin{array}{c}\text { Oral } \\
\text { (mandible) }\end{array}$ & $\begin{array}{l}\text { Basosquamous } \\
\text { carcinoma }\end{array}$ & Unknown & Yes $^{\dagger}$ & 2.6 \\
\hline \multirow[t]{2}{*}{7} & Pug & 8 & 11.6 & Ear & Mast cell tumor & Unknown & No & 0.5 \\
\hline & & & & Eyelid & Mast cell tumor & Unknown & No & 0.5 \\
\hline 8 & Cross breed & 10 & 5.0 & Oral (lip) & Mast cell tumor & Unknown & No & 0.1 \\
\hline 9 & Bull Terrier & 6 & 15.1 & Abdomen & Mast cell tumor & S.C Ml 5/10 HPF & No & 80 \\
\hline 10 & $\begin{array}{l}\text { Labrador } \\
\text { Retriever }\end{array}$ & 10 & 42.0 & Intranasal & $\begin{array}{l}\text { Squamous cell } \\
\text { carcinoma }\end{array}$ & Unknown & No & 2.0 \\
\hline
\end{tabular}

${ }^{\dagger}$ For patients no. 5 and 6 , the surgery prior to FLASH-RT was performed with unclean margins and there was local recurrence prior to initiating FLASH-RT.

disease. Possible adverse events were graded using the Veterinary Radiation Therapy Oncology Group (VRTOG) grading scheme for adverse events following radiotherapy (22). If the observed toxicity was found to be low-grade and well tolerated at followup, dose escalation to the subsequent patients was considered, taking the properties of the surrounding normal tissues into account.

\section{Additional Therapy}

Patients already on treatment with NSAIDs for arthritic disease, continued this treatment throughout the study period. Canine cancer patients with gross mast cell tumors were treated with antihistamines and or prednisolone for approximately one week before and after radiotherapy to reduce the risk of anaphylaxis or oedema related to mast cell degranulation. Patients receiving radiotherapy to the eyelid were treated with artificial teardrops after radiotherapy to increase lubrication of the eye. One patient (patient no. 8) with unilateral submandibular lymph node metastasis diagnosed prior to radiotherapy underwent surgery to remove the affected lymph node three weeks after radiotherapy. This patients went on to receive adjuvant chemotherapy to reduce risk of further metastasis. Patient no. 5 had the irradiated eye surgically removed one month after the second dose of radiotherapy. Patient no. 9 was started on oral therapy with a tyrosine kinase inhibitor one month after radiotherapy.

\section{Ethics}

Owners were asked to sign an informed written consent form, prior to enrollment of their animal in the study. The study was approved by the Local Ethical and Administrative Committee at Department of Veterinary Clinical Sciences, University of Copenhagen, the Danish Experimental Animals Inspectorate (2020-15-0201-00429) and the Swedish Board of Agriculture (reference number 5.2.18-02830/2020).

\section{RESULTS}

\section{Beam Characteristics}

The dose profiles and the measured depth dose curves demonstrated the typical characteristics of electron beams, with a high surface dose and a rapid drop in dose beyond dose maximum (Figure 3). The measured dose maximums, $R_{50^{-}}$ values, $\mathrm{R}_{80}$-values all increased with increasing field size, up to a field size of $\varnothing=4 \mathrm{~cm}$, after which the values were not further increased. The therapeutic range $\left(\mathrm{R}_{80}\right.$-value $)$ and half value depth $\left(\mathrm{R}_{50}\right.$-value) were $2.3 \mathrm{~cm}$ and $3.1 \mathrm{~cm}$, respectively, for the smallest field size $(\varnothing=2 \mathrm{~cm})$, and $3.1 \mathrm{~cm}$ and $3.8 \mathrm{~cm}$ for the largest field size $\left(10 \times 10 \mathrm{~cm}^{2}\right)$. The FWHMs and penumbra widths at $2 \mathrm{~cm}$ depth increased with increasing field size, ranging from 2.0 to $10.3 \mathrm{~cm}$ and from 0.1 to $1.1 \mathrm{~cm}$, respectively.

\section{Treatment Parameters}

The prescribed dose for the ten patients ranged from 15 to $35 \mathrm{~Gy}$ at dose maximum, depending on tumor type, tumor size, macroscopic/microscopic disease, previously published information $(5,9)$, and experience from prior patient treatments. The smallest field size used for treatment was a circular field with a diameter of $2 \mathrm{~cm}$, and the largest was a rectangular field of $8 \times 4 \mathrm{~cm}^{2}$ (Table 2). Two patients (no. 4 and 7) were irradiated at two tumor sites and one patient (no. 5) was reirradiated one month after the first treatment, which meant that a total of 13 doses were administered during the study period. Based on the Farmer-type ion-chamber signal, 92\% (12/13) of the treatments were measured to be within $5 \%$ of the prescribed dose. This was subsequently confirmed by the film based in vivo dosimetry, showing an average agreement between prescribed and delivered skin dose of $-1.8 \%$ (range $-9.4 \%$ to $+5.0 \%$ ). Average dose rates ranged between 400-500 Gy/s and treatments were delivered in 7-16 pulses corresponding to a total treatment time ranging from $30 \mathrm{~ms}$ to $75 \mathrm{~ms}$ (Table 2). The instantaneous dose rates, i.e., the dose rate within each pulse, were $\sim 7 \cdot 10^{5} \mathrm{~Gy} / \mathrm{s}$. 

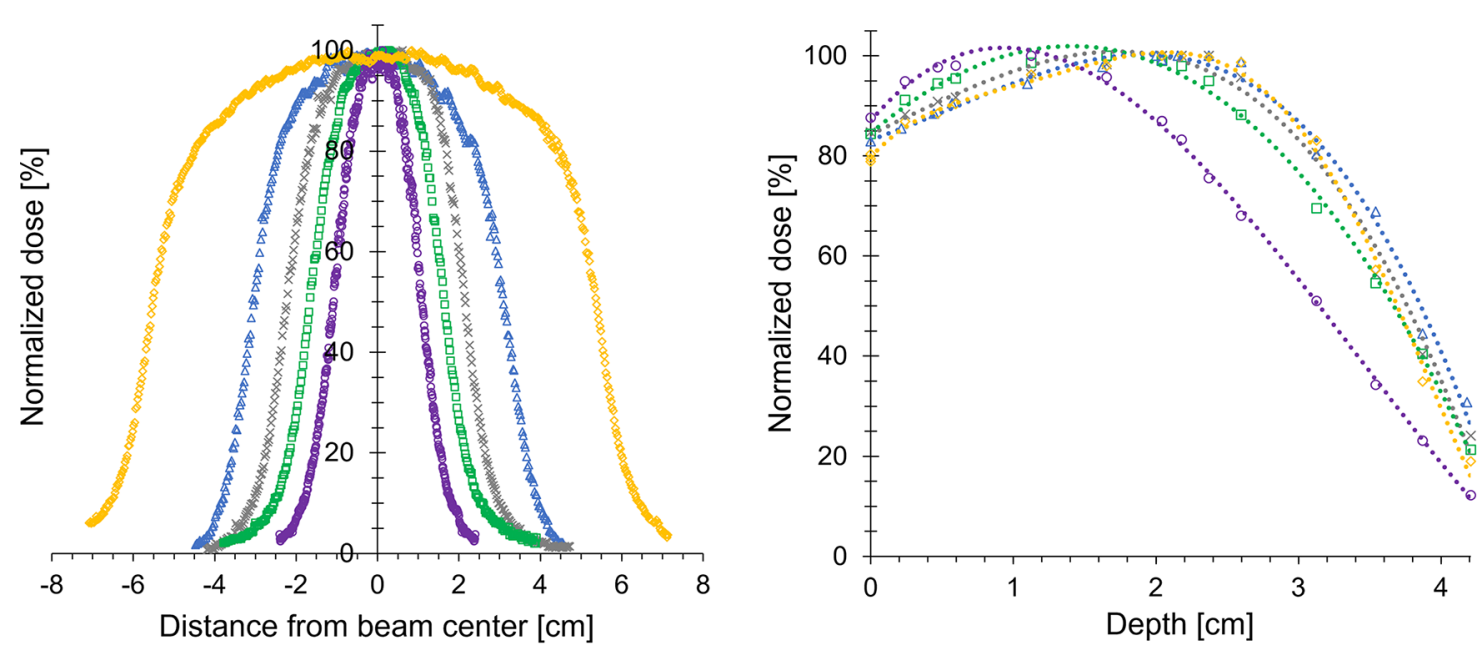

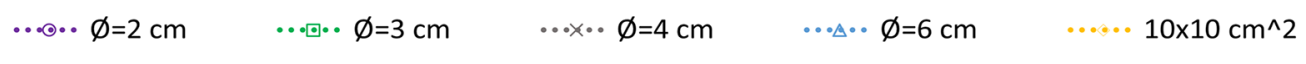

FIGURE 3 | Dose profiles at $2 \mathrm{~cm}$ (left panel) and percentage depth dose curves (right panel) measured in a solid water phantom, for various field sizes of the Cerrobend plates fitted in the electron applicator. The therapeutic range $\left(R_{80}\right.$-value) ranged from $2.3-3.2 \mathrm{~cm}$, depending on field size.

\section{Follow-Up Evaluation}

In general, observed adverse events were mild and consisted of local alopecia, leukotricia (whiteness of the fur), dry desquamation, mild erythema or swelling (Table 3). However, patient no. 10, which received a $35 \mathrm{~Gy}$ dose to the nasal planum, had a moist desquamation affecting the part of the nasal planum included in the radiation field (Figure 4). This was assessed as a grade 3 skin adverse event. The desquamation started approximately 14 days after the initial therapy and had resolved completely at 1 month post radiotherapy. The irradiated skin adjacent to the nasal planum only

TABLE 2 | Treatment parameters for the ten canine patients.

\begin{tabular}{|c|c|c|c|c|c|c|c|}
\hline $\begin{array}{l}\text { Patient } \\
\text { no. }\end{array}$ & $\begin{array}{l}\text { Field } \\
\text { size }\end{array}$ & $\begin{array}{l}\text { Bolus } \\
{[\mathrm{cm}]}\end{array}$ & $\begin{array}{l}\text { Est. volume receiving } \geq 80 \% \text { of prescribed } \\
\text { dose }\left[\mathrm{cm}^{3}\right]\end{array}$ & $\begin{array}{l}\text { Prescribed dose } \\
{[\text { [Gy] }}\end{array}$ & $\begin{array}{l}\text { Number of } \\
\text { pulses }\end{array}$ & $\begin{array}{l}\text { Treatment time } \\
{[\mathrm{ms}]}\end{array}$ & $\begin{array}{l}\text { Average dose rate } \\
{[\mathrm{Gy} / \mathrm{s}]}\end{array}$ \\
\hline 1 & $\begin{array}{l}8 \times 4 \\
\mathrm{~cm}^{2}\end{array}$ & 1.0 & 48 & 15 & 7 & 30 & 500 \\
\hline 2 & $\begin{array}{l}6 \times 2 \\
\mathrm{~cm}^{2}\end{array}$ & 1.5 & 8.1 & 15 & 8 & 35 & 430 \\
\hline 3 & $\begin{array}{l}6 \times 4 \\
\mathrm{~cm}^{2}\end{array}$ & 1.5 & 20 & 20 & 10 & 45 & 440 \\
\hline 4 & $\begin{array}{l}\varnothing=5 \\
\mathrm{~cm}\end{array}$ & 1.0 & 34 & 25 & 12 & 55 & 450 \\
\hline \multirow[t]{2}{*}{5} & $\begin{array}{l}\varnothing=3 \\
\mathrm{~cm}\end{array}$ & 1.5 & 4.3 & 25 & 12 & 55 & 450 \\
\hline & $\begin{array}{c}\varnothing=3 \\
\mathrm{~cm}\end{array}$ & 1.5 & 4.3 & $20^{+}$ & 9 & 40 & 500 \\
\hline & $\begin{array}{c}\varnothing=2 \\
\mathrm{~cm}\end{array}$ & 1.0 & 1.9 & 30 & 15 & 70 & 430 \\
\hline 8 & $\begin{array}{c}\varnothing=2 \\
\mathrm{~cm}\end{array}$ & 1.0 & 1.9 & 30 & 15 & 70 & 430 \\
\hline 9 & $\begin{array}{l}\varnothing=5 \\
\mathrm{~cm}\end{array}$ & 0 & 47 & 35 & 16 & 75 & 470 \\
\hline 10 & $\begin{array}{l}5 \times 2 \\
\mathrm{~cm}^{2}\end{array}$ & 0 & 13 & 35 & 16 & 75 & 470 \\
\hline
\end{tabular}

${ }^{\dagger}$ Patient no. 5 was re-irradiated approximately 4 weeks after the first treatment. 
TABLE 3 | Adverse events (in general mild cases of local alopecia, leukotricia, dry desquamation, mild erythema or swelling) graded using the VRTOG grading scheme for adverse events following radiotherapy.

\begin{tabular}{lccc}
\hline Patient no. & $\mathbf{7}$ days & 1 month & 3 months \\
\hline 1 & 0 & 1 & 1 \\
2 & 0 & $1^{\dagger}$ & 0 \\
3 & 0 & 1 & 1 \\
4 & 0 & 1 & 1 \\
& 0 & 1 & 1 \\
5 & 0 & 1 & 1 \\
& $n / a$ & $n / a$ & $n / a$ \\
6 & 0 & 1 & 1 \\
7 & 0 & 1 & 1 \\
& 0 & 1 & 1 \\
9 & 0 & 1 & 1 \\
10 & 0 & $3^{\dagger \dagger}$ & $3^{\dagger \dagger}$ \\
\hline
\end{tabular}

${ }^{\dagger}$ Patient no. 2 developed a small ulcer which was thought to be a suture reaction from previous surgery.

${ }^{t+}$ Patient no. 10 developed moist desquamation of the part of the nasal planum included in the radiation field.

showed evidence of mild grade 1 adverse events in terms of alopecia and mild dry desquamation. This patient received topical therapy with fucidic acid to reduce the risk of infection in the exposed dermis. Another patient (patient no. 2) developed a small ulcer in the treatment field, which was thought to be a suture reaction from previous surgery but could not be excluded as a grade 3 adverse event. This resolved with no further treatment.

The efficacy of the treatment during the follow-up period is summarized in Table 4. For patients with microscopic disease, no recurrence was observed during the study period. For 5/7 of the patients with solid tumors, the treatment resulted in stable disease or partial response after 1 month. Patient no. 7 showed a complete response in both tumors 3 months post irradiation. For patient no. 10 the tumor was located intranasally and response was evaluated based on clinical improvement of nasal airflow before and after therapy and visual inspection of the affected nostril. Whether the tumor response was partial or complete could not be determined clinically. Two patients (patient no. 5 and 9) had clear progressive disease after initially showing partial response to the treatment. For patient no. 7 and 8 exact measurements of tumor response were estimated partly based on clinical and visual examination rather than caliper measurement due to small size and subcutaneous or mucosal localization of tumors.

\section{DISCUSSION}

With the setup and dosimetric procedures described in this paper, initial experience of clinical FLASH-RT in canine cancer
7 days post FLASH-RT
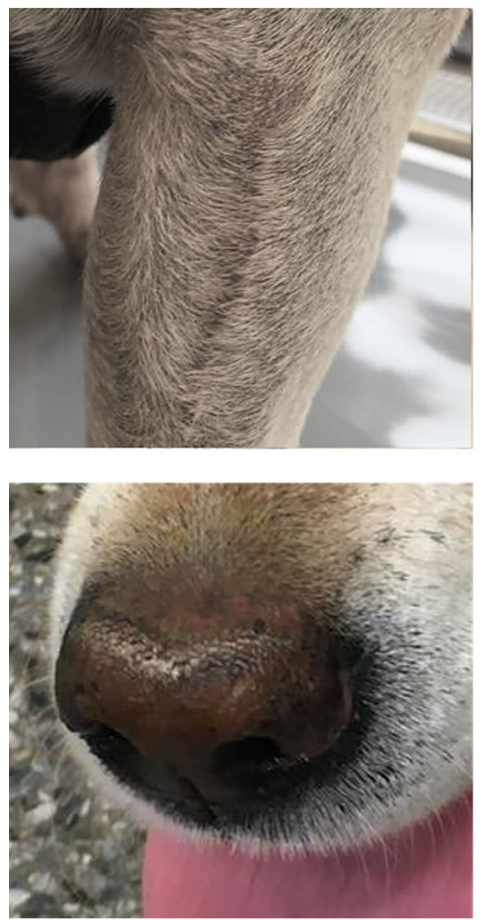

1 month post FLASH-RT
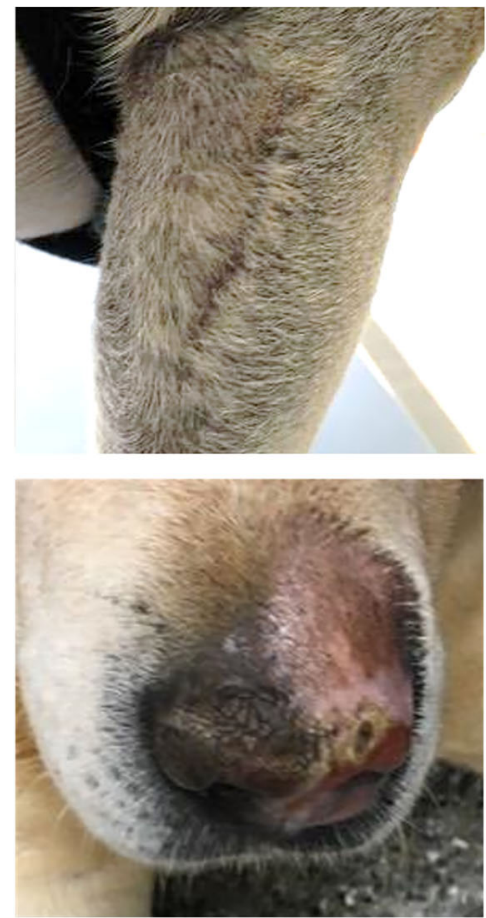

3 months post FLASH-RT
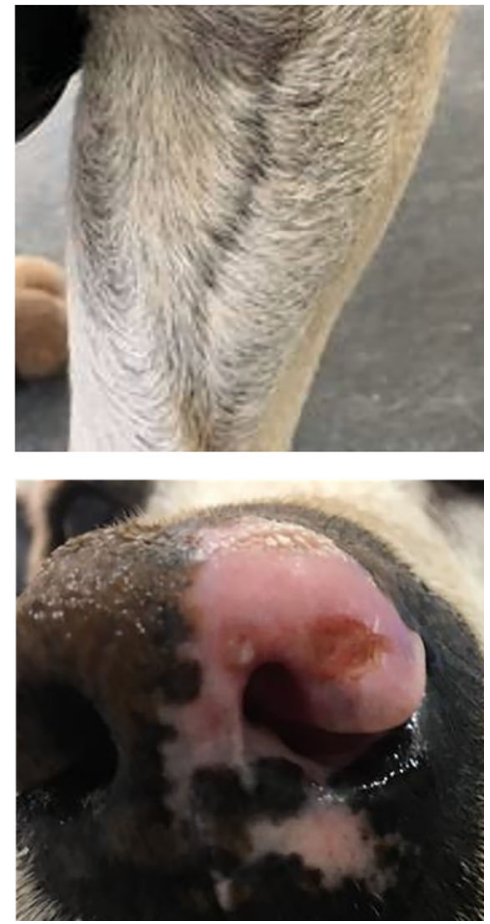

FIGURE 4 | Photographs of patient no. 1 with post-operative microscopic residual disease on the front limb (top row) and patient no. 10 with intranasal squamous cell carcinoma (bottom row) at 7 days, 1 month, and 3 months post FLASH-RT. 
TABLE 4 | Tumor response at 7 days, 1 month and 3 months post FLASH-RT, estimated based on the veterinary RECIST 1.0 criteria for patients with solid tumors, and disease-free interval for patients with microscopic disease.

\begin{tabular}{lccc}
\hline Patient no. & 7 days & 1 month & 3 months \\
\hline 1 & $\mathrm{NR}$ & $\mathrm{NR}$ & $\mathrm{NR}$ \\
2 & $\mathrm{NR}$ & $\mathrm{NR}$ & $\mathrm{NR}$ \\
3 & $\mathrm{NR}$ & $\mathrm{NR}$ & $\mathrm{NR}$ \\
4 & $\mathrm{SD}$ & $\mathrm{SD}$ & $\mathrm{PR}$ \\
& $\mathrm{SD}$ & $\mathrm{SD}$ & $\mathrm{SD}$ \\
5 & $\mathrm{SD}$ & $\mathrm{PD}$ & $\mathrm{n} / \mathrm{a}$ \\
& $\mathrm{SD}$ & $\mathrm{PD}$ & $\mathrm{n} / \mathrm{a}$ \\
6 & $\mathrm{SD}$ & $\mathrm{PR}$ & $\mathrm{PR}$ \\
7 & $\mathrm{SD}$ & $\mathrm{PR}$ & $\mathrm{CR}$ \\
& $\mathrm{SD}$ & $\mathrm{PR}$ & $\mathrm{CR}$ \\
8 & $\mathrm{PR}$ & $\mathrm{PR}$ & $\mathrm{PR}$ \\
9 & $\mathrm{PR}$ & $\mathrm{PD}$ & $\mathrm{PD}$ \\
10 & $\mathrm{SD}$ & $\mathrm{PR} / \mathrm{CR}{ }^{\dagger+}$ & $\mathrm{CR}^{\dagger \dagger}$ \\
\hline
\end{tabular}

$R$, Recurrence; NR, No recurrence; $S D$, Stable disease; $P R$, Partial response; $C R$, Complete response; PD, Progressive disease; $n / a$, Not applicable.

${ }^{\dagger}$ The patient showed PR at 14 days followed by PD at 4 weeks after both the first and second treatment. At 4 weeks after the second treatment the tumor was surgically resected.

${ }^{{ }^{t \dagger}}$ The tumor response for patient no. 10 was based on clinical response and evaluation of nasal airflow.

patients using a modified clinical linear accelerator is presented. The measured depth dose curves showed that the beam can deliver $>80 \%$ of the prescribed dose to a volume along the central axis ranging from the skin surface to a depth of $2-3 \mathrm{~cm}$ in tissue, after which the dose drops off sharply due to scattering and energy loss. These features make the beam suitable for treatments of superficial or subcutaneous tumors, but of limited use for deeper seated tumors. Hence, only superficial and subcutaneous tumors were included in this trial.

As the ten canine cancer patients included in this study were the first patients treated with FLASH-RT at our clinical accelerator, we started at a dose level that was considered as safe based on previously published information $(5,9)$. For one of the patients that initially showed partial response (patient no. 5), the dose administered was likely too low to control the cancer growth and hence progressive disease was seen at 4 weeks post treatment. For the second patient showing progressive disease 4 weeks post treatment (patient no. 9), the initial tumor was multilobulated and part of the tumor was seated in the abdominal musculature reaching a depth of $4.1 \mathrm{~cm}$, hence it is likely that the deeper part of the tumor only received a limited part of the prescribed dose. The normal tissues showed a good tolerance during the follow-up period when irradiated with high single doses of FLASH-RT, also for the patients given 30 and 35 Gy, where mainly mild or moderate transient adverse events were observed, indicating possible further opportunities for dose escalation and extended margins to enhance the probability of tumor control. The mucous membranes in the oral cavity are generally sensitive to acute radiation side effects which can have impact on the patients' appetite and ability to eat and have negative effect on quality of life (23). In the current study we found that FLASH-RT to the oral cavity was well tolerated and side effects were limited to grade 1 early and late side effects in terms of light injection of the mucous membranes and alteration in pigmentation. This suggests that this single fraction treatment modality can be applied to treat oropharyngeal tumors without negative impact on quality of life. This initial feasibility and safety study, with a small and heterogeneous group of participants, was not designed to provide statistically verifiable results of tumor response to FLASH-RT. A larger study is required to investigate statistical significance for the therapeutic benefit for specific cancer types and different dose levels. In addition, to investigate whether FLASH-RT is superior in sparing normal tissue compared to conventional radiotherapy, with equally effective tumor control, a comparative randomized trial with both modalities would be required in a more homogeneous patient group.

A limitation of this study is the lack of a treatment planning system for the FLASH beam to display the dose distribution in the patients. Instead, the treatment volumes were estimated based on vertical $2 \mathrm{D}$ film measurements in a phantom, and presented together with the estimated gross tumor volume. Using the in vivo film measurements, we could confirm that the prescribed dose was delivered to the patient surface with an agreement within $10 \%$. Complex treatment geometries, such as tissue inhomogeneities, uneven air gaps and sloping surfaces, made it difficult to predict the dose distribution in advance. The ideal situation, with the electron beam impinging along the normal towards a flat surface of a homogeneous tissue, was not fulfilled in the treatments, which may have led to degrading of the dose distribution estimated to be less than $10 \%$. Ion-chambers, which are the standard real time dosimeter in conventional radiotherapy, experience a large drop in ion collection efficiency at the ultra-high dose rates associated with FLASH radiation (24-26), making them imprecise and impractical for real time dose measurements. Therefore, the Farmer-type ion-chamber used in the current setup was functioning solely as an output monitor. Approaching human clinical trials, novel dosimetric procedures that ensure accurate delivery of the prescribed dose at FLASH irradiations, by real time dose measurements, are required. We have previously shown that the ion collection efficiency in a built-in monitor chamber can be increased by increasing the polarizing voltage over the chamber (25), and we are currently working on employing this knowledge for the setup used for our companion animal treatments by using an external monitor chamber positioned at the top of the electron applicator. We believe this approach will bring clinical dosimetry in FLASH up to the standards of conventional radiotherapy. In addition, human clinical studies will require a redundant safety system, which is an added technical challenge when using a clinical linear accelerator for FLASH-RT. When the accelerator is operated in FLASH mode, interruption of the electron beam after delivery of the desired number of pulses, is solely dependent on a diode placed in the radiation field functioning as a pulse counter. To further increase the safety during FLASH delivery, we are working on a solution where the two independent channels in the external monitor chamber can be used to interrupt the beam, similar to the method used for controlling the dose delivery in conventional radiotherapy. Furthermore, it would be favorable to be able to adjust the electron beam energy depending on the depth and size of the tumor. Currently, our FLASH beam is limited to a single energy 
of $10 \mathrm{MeV}$, although attempts to adjust the energy is ongoing. This would allow us to choose the treatment depth by applying an appropriate energy, and thus better exploit the advantages of an electron beam.

In addition to the companion animal cancer patients receiving superior treatment and providing valuable experience in setting up a clinical workflow for human treatments, companion animal cancer patient studies have the potential to greatly inform radiobiology studies. The mechanisms behind the FLASH sparing effect are yet to be fully understood, but the main hypothesis so far is oxygen depletion. We have previously shown in vitro that the FLASH effect depends on oxygen concentration (27). Due to the similarities between the tumors of companion animals and humans, also in terms of oxygen profiles (19), canine cancer patients provide an opportunity for further studying the oxygen dependence in a clinically relevant setting.

In conclusion, the first experience of electron FLASH-RT in canine cancer patients in a clinical setting is presented. Treatments were found to be feasible, with partial response, complete response or stable disease recorded in 11/13 irradiated tumors. Adverse events observed at follow-up ranging from 3-6 months were mild and consisted of local alopecia, leukotricia, dry desquamation, mild erythema or swelling. Only one patient receiving a 35 Gy dose to the nasal planum, had a grade 3 skin adverse event. Dosimetric procedures, safety and an efficient clinical workflow for FLASH-RT was established. The experience from this initial trial, in terms of a safe and efficient workflow for FLASH-RT in a clinical setting, will be used as a basis for a veterinary phase I/II clinical trial with more specific patient inclusion selection, and subsequently for human trials.

\section{DATA AVAILABILITY STATEMENT}

The original contributions presented in the study are included in the article/supplementary material. Further inquiries can be directed to the corresponding author.

\section{REFERENCES}

1. Favaudon V, Caplier L, Monceau V, Pouzoulet F, Sayarath M, Fouillade C, et al. Ultrahigh Dose-Rate FLASH Irradiation Increases the Differential Response Between Normal and Tumor Tissue in Mice. Sci Trans Med (2014) 6(245):245ra93. doi: 10.1126/scitranslmed.3008973

2. Montay-Gruel P, Petersson K, Jaccard M, Boivin G, Germond JF, Petit B, et al. Irradiation in a Flash: Unique Sparing of Memory in Mice After Whole Brain Irradiation With Dose Rates Above 100Gy/S. Radiother Oncol (2017) 124 (3):365-9. doi: 10.1016/j.radonc.2017.05.003

3. Montay-Gruel P, Acharya MM, Petersson K, Alikhani L, Yakkala C, Allen BD, et al. Long-Term Neurocognitive Benefits of FLASH Radiotherapy Driven by Reduced Reactive Oxygen Species. Proc Natl Acad Sci USA (2019) 116:1094351. doi: 10.1073/pnas.1901777116

4. Fouillade C, Curras-Alonso S, Giuranno L, Quelennec E, Heinrich S, BonnetBoissinot S, et al. FLASH Irradiation Spares Lung Progenitor Cells and Limits the Incidence of Radio-Induced Senescence. Clin Cancer Res (2019) 26 (6):1497-506. doi: 10.1158/1078-0432.CCR-19-1440

5. Vozenin MC, De Fornel P, Petersson K, Favaudon V, Jaccard M, Germond JF, et al. The Advantage of FLASH Radiotherapy Confirmed in Mini-Pig and

\section{ETHICS STATEMENT}

The animal study was reviewed and approved by Local Ethical and Administrative Committee at Department of Veterinary Clinical Sciences, University of Copenhagen, the Danish Experimental Animals Inspectorate (2020-15-0201-00429), and the Swedish Board of Agriculture (reference number 5.2.18-02830/2020). Written informed consent was obtained from the owners for the participation of their animals in this study.

\section{AUTHOR CONTRIBUTIONS}

All authors contributed to the study design. EK, MA, KB, CC, $\mathrm{AH}$, and $\mathrm{KP}$ administered the treatments and collected the data. EK drafted the manuscript, with support from MA. All authors contributed to the article and approved the submitted version.

\section{FUNDING}

This work was supported by Mrs Berta Kamprad Foundation, The Swedish Cancer Society and Skåne University Hospital's foundations and donations.

\section{ACKNOWLEDGMENTS}

The authors wish to thank HannaMaria Thomasson for assisting us with the treatments, and Lars Andersson Ljus and Michael Lempart for providing engineering solutions. All three are affiliated at the Department of Hematology, Oncology and Radiation Physics, Skåne University Hospital, Lund, Sweden.

Cat-Cancer Patients. Clin Cancer Res (2019) 25:35-42. doi: 10.1158/1078 0432.CCR-17-3375

6. Levy K, Natarajan S, Wang J, Chow S, Eggold JT, Loo P, et al. Abdominal FLASH Irradiation Reduces Radiation-Induced Gastrointestinal Toxicity for the Treatment of Ovarian Cancer in Mice. Sci Rep (2020) 10(1):21600. doi: 10.1038/s41598-020-78017-7

7. Bourhis J, Montay-Gruel P, Gonçalves Jorge P, Bailat C, Petit B, Ollivier J, et al. Clinical Translation of FLASH Radiotherapy: Why and How? Radiother Oncol (2019) 139:11-7. doi: 10.1016/j.radonc.2019.04.008

8. Montay-Gruel P, Acharya MM, Jorge PG, Petit B, Petridis IG, Fuchs P, et al. Hypofractionated FLASH-RT as an Effective Treatment Against Glioblastoma That Reduces Neurocognitive Side Effects in Mice. Clin Cancer Res (2021) 27 (3):775-84. doi: 10.1158/1078-0432.CCR-20-0894

9. Bourhis J, Sozzi WJ, Jorge PG, Gaide O, Bailat C, Duclos F, et al. Treatment of a First Patient With FLASH-Radiotherapy. Radiother Oncol (2019) 139:1822. doi: 10.1016/j.radonc.2019.06.019

10. Harrington KJ. Ultrahigh Dose-Rate Radiotherapy: Next Steps for FLASHRT. Clin Cancer Res (2019) 25:3-5. doi: 10.1158/1078-0432.CCR-18-1796

11. Wilson JD, Hammond EM, Higgins GS, Petersson K. Ultra-High Dose Rate (FLASH) Radiotherapy: Silver Bullet or Fool's Gold? [Published Correction 
Appears in Front Oncol. 2020 Feb 25;10:210]. Front Oncol (2020) 9:1563. doi: 10.3389/fonc. 2019.01563

12. Jaccard M, Durán MT, Petersson K, Germond JF, Liger P, Vozenin MC, et al. High Dose-Per-Pulse Electron Beam Dosimetry: Commissioning of the Oriatron Ert6 Prototype Linear Accelerator for Preclinical Use. Med Phys (2018) 45:863-74. doi: 10.1002/mp.12713

13. Schüler E, Trovati S, King G, Lartey F, Rafat M, Villegas M, et al. Experimental Platform for Ultra-High Dose Rate FLASH Irradiation of Small Animals Using a Clinical Linear Accelerator. Int J Radiat Oncol Biol Phys (2017) 97:195-203. doi: 10.1016/j.ijrobp.2016.09.018

14. Lempart M, Blad B, Adrian G, Bäck S, Knöös T, Ceberg C, et al. Modifying a Clinical Linear Accelerator for Delivery of Ultra-High Dose Rate Irradiation. Radiother Oncol (2019) 139:40-5. doi: 10.1016/j.radonc.2019.01.031

15. Rahman M, Ashraf MR, Zhang R, Bruza P, Dexter CA, Thompson L, et al. Electron FLASH Delivery At Treatment Room Isocenter for Efficient Reversible Conversion of a Clinical LINAC. Int J Radiat Oncol Biol Phys (2021) S0360-3016(21):00024-9. doi: 10.1016/j.ijrobp.2021.01.011

16. McEntee MC. Veterinary Radiation Therapy: Review and Current State of the Art. J Am Anim Hosp Assoc (2006) 42(2):94-109. doi: 10.5326/ 0420094

17. Paoloni M, Khanna C. Translation of New Cancer Treatments From Pet Dogs to Humans. Nat Rev Cancer (2008) 8(2):147-56. doi: 10.1038/nrc2273

18. Rowell JL, McCarthy DO, Alvarez CE. Dog Models of Naturally Occurring Cancer. Trends Mol Med (2011) 17(7):380-88. doi: 10.1016/j.molmed. 2011.02.004

19. Nolan MW, Kent MS, Boss MK. Emerging Translational Opportunities in Comparative Oncology With Companion Canine Cancers: Radiation Oncology. Front Oncol (2019) 9:1291. doi: 10.3389/fonc.2019.01291

20. Jaccard M, Petersson K, Buchillier T, Germond JF, Durán MT, Vozenin MC, et al. High Dose-Per-Pulse Electron Beam Dosimetry: Usability and DoseRate Independence of EBT3 Gafchromic Films. Med Phys (2017) 44(2):72535. doi: $10.1002 / \mathrm{mp} .12066$

21. Nguyen SM, Thamm DH, Vail DM, London CA. Response Evaluation Criteria for Solid Tumours in Dogs (V1.0): A Veterinary Cooperative
Oncology Group (VCOG) Consensus Document. Vet Comp Oncol (2015) 13(3):176-83. doi: $10.1111 /$ vco.12032

22. Ladue T, Klein MK. Toxicity Criteria of The Veterinary Radiation Therapy Oncology Group. Vet Radiol Ultrasound (2001) 42(5):475-6. doi: 10.1111/j.1740-8261.2001.tb00973.x

23. Collen EB, Mayer MN. Acute Oropharyngeal Effects of Full-Course Radiation Treatment of Tumors of the Head. Can Vet J (2008) 49:509-12.

24. Petersson K, Jaccard M, Germond JF, Buchillier T, Bochud F, Bourhis J, et al. High Dose-Per-Pulse Electron Beam Dosimetry - a Model to Correct for the Ion Recombination in the Advanced Markus Ionization Chamber. Med Phys (2017) 44(3):1157-67. doi: 10.1002/mp.12111

25. Konradsson E, Ceberg C, Lempart M, Blad B, Bäck S, Knöös T, et al. Correction for Ion Recombination in a Built-in Monitor Chamber of a Clinical Linear Accelerator At Ultra-High Dose Rates. Radiat Res (2020) 194(6):580-86. doi: 10.1667/RADE-19-00012

26. Ashraf MR, Rahman M, Zhang R, Williams BB, Gladstone DJ, Pogue BW, et al. Dosimetry for FLASH Radiotherapy: A Review of Tools and the Role of Radioluminescence and Cherenkov Emission. Front Phys (2020) 8:328. doi: $10.3389 /$ fphy.2020.00328

27. Adrian G, Konradsson E, Lempart M, Bäck S, Ceberg C, Petersson K. The FLASH Effect Depends on Oxygen Concentration. Br J radiology. (2020) 93 (1106):20190702. doi: 10.1259/bjr.20190702

Conflict of Interest: The authors declare that the research was conducted in the absence of any commercial or financial relationships that could be construed as a potential conflict of interest.

Copyright (๑ 2021 Konradsson, Arendt, Bastholm Jensen, Børresen, Hansen, Bäck, Kristensen, Munck af Rosenschöld, Ceberg and Petersson. This is an open-access article distributed under the terms of the Creative Commons Attribution License (CC BY). The use, distribution or reproduction in other forums is permitted, provided the original author(s) and the copyright owner(s) are credited and that the original publication in this journal is cited, in accordance with accepted academic practice. No use, distribution or reproduction is permitted which does not comply with these terms. 